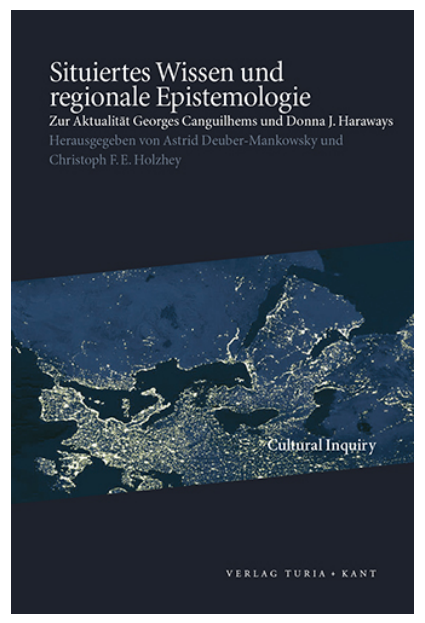

Situiertes Wissen und regionale Epistemologie: Zur Aktualität Georges Canguilhems und Donna J. Haraways, hg. v. Astrid Deuber-Mankowsky und Christoph F. E. Holzhey, Cultural Inquiry, 7 (Wien: Turia + Kant, 2013), S. 141-59

\section{MARIA MUHLE (1D}

\section{Vom Vitalen zum Sozialen Überlegungen zu einem politischen Wissen im Anschluss an Canguilhem}

\section{ZITIERVORGABE:}

Maria Muhle, »Vom Vitalen zum Sozialen: Überlegungen zu einem politischen Wissen im Anschluss an Canguilhem «, in Situiertes Wissen und regionale Epistemologie: Zur Aktualität Georges Canguilhems und Donna J. Haraways, hg. v. Astrid Deuber-Mankowsky und Christoph F. E. Holzhey, Cultural Inquiry, 7 (Wien: Turia + Kant, 2013), S. 141-59<https://doi.org/10.37050/ci-07_07>

\section{ANGABE ZU DEN RECHTEN:}

(C) by the author(s)

This version is licensed under a Creative Commons AttributionShareAlike 4.0 International License. 


\title{
VOM VITALEN ZUM SOZIALEN
}

\author{
Überlegungen zu einem politischen Wissen \\ im Anschluss an Canguilhem
}

Maria Muhle

In seinen Neuen Überlegungen zum Normalen und Pathologischen, die als Nachtrag zum 1943 vollendeten Versuch über einige Probleme, das Normale und das Pathologische betreffend "zwanzig Jahre später « erscheinen, stellt Georges Canguilhem die Frage nach der Verfasstheit sozialer Normen vor dem Hintergrund seiner Untersuchungen zum Begriff des Lebens und der vitalen Norm. Canguilhem, der hier auch die Arbeiten Michel Foucaults - besonders Die Geburt der Klinik - rezipiert, hält jedoch fest, dass er die soziale Norm einzig zum besseren Verständnis des Organismus untersucht: »Zum besseren Verständnis des Organismus erlaube ich mir einige Abstecher in die Gesellschaft. « ${ }^{1}$

Im Folgenden möchte ich ausgehend von dieser Feststellung, aber abweichend von Canguilhem, die Frage nach dem Vitalen zum besseren Verständnis der Gesellschaft - also des Sozialen - untersuchen. Meine These ist, dass es gerade das notwendige Ineinandergreifen, die Synthese bzw. das Mischverhältnis von sozialen und vitalen Normen sind, die Aufschluss geben über die Beschaffenheit des Sozialen. Im Mittelpunkt der Analyse steht dementsprechend zunächst Canguilhems Verständnis sozialer Normen sowie ihr problematischer und werkintern variierender Bezug zu den vitalen Normen: In seinem Versuch wie auch in der Aufsatzsammlung Die Erkenntnis des Lebens ${ }^{2}$ hat Canguilhem die Norm stets unter dem Vorzeichen der Normativität, also ihrer inneren Dynamik, und hinsichtlich des Lebendigen im Allgemeinen untersucht. Canguilhems explizites Vorhaben war es hier, die vitale Norm fern von sozialen Einflüssen als reines Phänomen des Lebendigen zu untersuchen, da jedweder soziale Einfluss das »medizinische Experiment « und dessen Gegenstand, das Leben, verfälschen musste. Diese Position wird in den Neuen Überlegungen dahingehend reformuliert, dass die sozialen Phänomene nun nicht mehr dem Vitalen bzw. den vitalen Normen nachgeordnet sind, sondern in zwei unabhängige Regimes, zwei "allures ", Gangarten oder Allüren des Lebens, die soziale und die vitale, zerfallen, die Canguilhem als inkommensurabel bezeichnet. Der Bezug dieser bei- 
den Regimes ist folglich kein hierarchischer mehr, in dem das Soziale nur als dem Vitalen nachgeordnet erfasst wird, vielmehr wird er nun von Canguilhem als ein mimetischer Bezug bezeichnet. Die doppelte Implikation dieser These - für den Begriff des Lebens einerseits und für die Frage nach den sozialen Normen andererseits - soll in diesem Text untersucht werden. Denn es ist gerade Canguilhems Rede von der Imitation oder Mimesis des Vitalen durch das Soziale, die, so meine Annahme, einen entscheidenden Impuls für Foucaults Ausformulierung der Biopolitik bzw. der Gouvernementalität als einer Macht bzw. Regierung des Lebens der Bevölkerung liefert.

\section{DIE POLARITÄT ALLES LEBENDIGEN}

Der Versuch über einige Probleme, das Normale und das Pathologische betreffend, den Canguilhem 1943 als seine Doktorarbeit der Medizin vorlegt, macht sich die Untersuchung der Grenzziehungen zwischen den normalen und pathologischen Phänomenen sowie der Implikationen derselben für den Begriff des Lebens zur Aufgabe. Das Nachdenken über die Krankheit bzw. über die Gesundheit als "Stille der Organe « (Leriche) wird hier nicht hinsichtlich der Medizin oder Biologie unternommen, sondern vielmehr gegen diese und mit Blick auf den Standpunkt des Kranken selbst, der im Gegensatz zur traditionellen medizinischen Auffassung von Canguilhem als nicht neutral verstanden wird: Auf die Widerlegung der positivistischen Auffassung, die pathologischen Phänomene seien einzig quantitative Variationen der normalen Phänomene, folgt die Untersuchung der Originalität des Pathologischen und der individuellen Erfahrung der Krankheit durch den Kranken, die sich in der Klinik (und nicht in der Physiologie als Wissenschaft) vollzieht. Während also der erste Teil des Versuchs die Vernachlässigung des biologischen Individuums in der Homogenisierung von Normalem und Pathologischem durch die wissenschaftliche Norm herausstellt und kritisiert, befasst sich der zweite Teil, »Gibt es Wissenschaften vom Normalen und Pathologischen? ", mit der Genese der organischen Individualität in und durch die Erfahrung der Krankheit. Denn es ist die Krankheit, die das Lebendige zum Lebendigen macht und zugleich das Bestreben eines jeden biologischen Individuums, am Leben zu bleiben und seine Kraft zu verstärken, saufdeckt .

Diese »Lehre « übernimmt Canguilhem, ähnlich wie Foucault später mit seiner Rede vom »Mortalismus « in der Geburt der Klinik, von 
Xavier Bichat, der in seinen Recherches physiologiques sur la vie et la mort von 1800 das Leben als »die Gesamtheit der Funktionen, die dem Tod widerstehen « bestimmt hatte. ${ }^{3}$ Damit wird die negative Dimension des Todes in das Leben derart eingetragen, dass eine ‘klassische< Verwissenschaftlichung des Lebens und die damit einhergehende Negation seiner spezifischen Dynamik nicht mehr haltbar scheinen: Der wissenschaftlichen Medizin oder Physiologie setzt Canguilhem ein Wissen vom Leben entgegen, das immer auch ein lebendiges Wissen ist, in dem Wissen und Leben sich gegenseitig durchdringen: »Der Verstand darf sich auf das Leben nur beziehen, wenn er die Originalität des Lebens anerkennt. Das Denken des Lebendigen muss die Idee des Lebendigen dem Lebendigen selbst entnehmen. «4

Diese Originalität des Lebens fasst Canguilhem in normativen Begriffen, die ihn zur Bestimmung einer spezifischen »Normativität " des Lebens führen. Das Leben ist normativ, wenn es die bestehenden Normen in Frage stellt und neue Normen schafft, wenn es also einem schöpferischen Impuls folgt. Das bedeutet, dass das Lebendige zugleich seine vitalen Werte, seine Normen und sein eigenes Milieu - und sich selbst in und durch diese - hervorbringt. So zeigt Canguilhem in »Das Lebendige und sein Milieu ", inwiefern das Leben gerade nicht in ein bestehendes (physikalisches) Milieu bineingeworfen wird, dem es in der Folge unterworfen wäre - so wie es selbst noch die klassisch animistischen Erklärungen des Lebens annahmen $-:^{5}$ »Ein Lebewesen unter experimentell konstituierten Bedingungen zu untersuchen bedeutet, ihm ein Milieu zu erschaffen, ihm ein Milieu aufzuzwingen. Das Besondere des Lebewesens besteht nun aber darin, sich selbst sein Milieu zu schaffen, es selbst auszubilden. ${ }^{6}$

Im Anschluss an Uexküll und Goldstein vernachlässigt Canguilhem hierbei den Unterschied zwischen menschlichem und tierischem Leben hinsichtlich der Untersuchung des Lebendigen im Allgemeinen, denn jedes Tier - paradigmatisch die Uexküllsche Zecke - verhält sich bei der Ausbildung seines Milieus im vitalen Sinne normativ, insofern es in der Lage ist, bestimmte (ihm förderliche) Elemente aus seiner Umgebung »zu bevorzugen « und andere (bedrohliche oder auch nur ihm gleichgültige Elemente) »auszuschließen [préférer et exclure] «:

[D]ie biologischen Funktionen, so wie die Beobachtung sie zeigt, [bleiben] unverständlich, wenn sie lediglich die verschiedenen Zustände einer gegenüber den Umweltveränderungen passiven Materie wiedergeben. In Wahrheit ist die Umwelt des Lebewesens immer auch dessen Werk, weil es sich 
stets bestimmten Einflüssen entzieht und anderen wiederum aussetzt. Von der Welt aller Lebewesen lässt sich sagen, was Reininger über die Welt des Menschen sagt: »Unser Weltbild ist immer zugleich ein Wertbild. ${ }^{7}$

Leben als vitale Normativität ist Sich-Aussetzen und Sich-Entziehen, oder, in den Worten Canguilhems: »Leben bedeutet schon für die Amöbe: auswählen und verwerfen. ${ }^{8}$ Ähnlich heißt es in dem späteren Text »Le concept et la vie«: »Leben, egal in welchem Maßstab, bedeutet auswählen und vernachlässigen. «9 Das Lebendige sucht sich jene Impulse aus seiner Umwelt, die ihm zugute kommen, und vermeidet zugleich jene anderen Impulse, Bedrohungen oder Grenzen, die sein Leben einschränken würden:

Dem Tier erscheint es einfacher, das zu tun, was es bevorzugt, was es privilegiert. Es hat seine eigenen vitalen Normen. Der Zusammenhang zwischen dem Lebendigen und dem Milieu etabliert sich als eine Auseinandersetzung*, in die das Lebewesen seine eigenen Normen der Beurteilung von Situationen mit einbringt, in der es das Milieu beherrscht und es sich anpasst. ${ }^{10}$

\section{DAS DOPPELTE LEBEN}

Das Leben wird also als Aktivität der Differenzierung beschrieben, die hier eine doppelte Bedeutung annimmt, nämlich die des Erhalts (durch Regulierung) und der Produktion oder Schöpfung (création), die auch als Individualisierung beschrieben wird. In diesem Sinne bezeichnet die Normativität zunächst die innere Autonomie des Organismus, d. h. das homöostatische Gleichgewicht der organischen Konstanten. ${ }^{11}$ Ein solches Verständnis von vitaler Normativität als Aufrechterhaltung des Gleichgewichts im milieu intérieur setzt zugleich ein holistisches Verständnis des Organismus voraus, wie Canguilhem es von Goldstein übernimmt. ${ }^{12}$ Der Organismus wird hier anhand der globalen Aktivität der Regulierung gedacht, die mit eben jener Möglichkeit der Aufrechterhaltung des inneren, organischen Gleichgewichts einhergeht. Zur Aufrechterhaltung dieses Gleichgewichts muss der Organismus all jene Faktoren ausschließen, die den funktionalen Wert beeinträchtigen könnten, von dem Canguilhem schreibt, dass »er als Norm in der Struktur selbst enthalten ist «; er tut dies über die Regulierung, die als »biologische Tatsache par excellence " gilt. $^{13}$ 
Zugleich scheint es, als befinde sich diese Form der Regulierung in gefährlicher Nähe zum pathologischen Zustand des Lebens, den Canguilhem, erneut mit Goldstein, nicht nur als ein von Außen durch das Milieu beherrschtes Leben beschrieben hat; vielmehr ist bereits dasjenige Leben, "das sich gegen etwas stellt", also ein reaktives Leben ein »bedrohtes Leben ${ }^{14}$ und die Regulierung somit die Antwort auf die Gefahr einer Außenbestimmung des Lebens. In Absetzung hierzu bezeichnet die Normativität bei Canguilhem in einem zweiten Sinne den ich als genuin vital bezeichnen möchte - die vollkommene Priorität des Lebendigen vor seinem Milieu, d. h. seine vollkommene Autonomie gegenüber äußeren Zwängen. In diesem zweiten Sinne ist die Normativität des Lebens ein schöpferischer oder schaffender Prozess, eine Dynamik, die den Vorgang der Individualisierung bzw. der Produktion von Individualität innerhalb des Lebendigen (und wiederum alles Lebendigen, nicht nur des menschlichen) bezeichnet: »Jedes Lebendige individualisiert sich durch die Werte, die es aktualisiert. « ${ }^{15}$

Das Lebendige be- und entwertet und schafft sich so neue, vitale Werte, die es ihm ermöglichen, seine spezifische Dynamik zu entfalten. Diese besteht darin, die erreichten Normen oder Werte immer wieder zu »riskieren « und aufs Spiel zu setzen, da das Lebendige sich sonst in einem künstlichen Gleichgewicht der Organfunktionen immobilisiert. Denn in einem solchen Gleichgewichtszustand (wie ihn z. B. das Labor künstlich herstellt) verliert das Leben die Fähigkeit (oder Kraft), seine alten Normen zu sprengen und sich, seine Normen und sein Milieu permanent neu zu schaffen. Das festgesetzte, nicht-dynamische Leben verliert den Luxus, krank werden zu können, da es sich zu seiner Krankheit nicht mehr normativ verhalten kann.

Folglich wird die vitale Normativität von Canguilhem gerade nicht als Anpassung gedacht, wie im Falle eines homöostatischen Systems, das Abweichungen integrieren und auf ein Normalmaß hin ausgleichen kann; vielmehr ist die vitale Normativität permanente Überschreitung und Infragestellung des Gegebenen: Ein Lebewesen verhält sich normativ, wenn es sich nicht an ein bestehendes Milieu oder eine bestehende Norm anpasst - dann wäre es pathologisch -, sondern sich sein eigenes Milieu und seine eigenen Normen schafft. Canguilhems normativer Lebensbegriff bildet sich in der Polarität zwischen diesen beiden Dimensionen aus, die untrennbar miteinander verbunden sind: Denn nur in seiner Abweichung von der Norm kann das Leben normativ sein, d. h. neue Normen produzieren, die ihrerseits auf eine neue, vorläufige Nor- 
malität ausgerichtet sind. Die Normativität besteht darin, »die geltenden Normen zu sprengen [faire craquer les normes] «. ${ }^{16}$ Erst vor dem Hintergrund einer solchen schöpferischen Kraft ist ein inneres, jedoch immer prekäres Gleichgewicht möglich: Die Normalität gründet auf der Normativität. In diesem Sinne schreibt Canguilhem den physiologischen Konstanten eine »vorwärtstreibende Kraft [valeur propulsive] « zu, die es dem Lebendigen erlaubt, sich normativ im genannten Sinne zu verhalten. Im Gegensatz dazu zeugt der pathologische Zustand »von der Zerbrechlichkeit des durch die Krankheit gesetzten Normalen. Die pathologischen Konstanten sind regressiv und streng auf Selbsterhaltung gerichtet. $" 17$

Die Herausforderung des Canguilhemschen Lebensbegriffs liegt also darin, dass sich die organische Normalität permanent den normativen Abweichungen ausgesetzt sieht, d. h. das Leben nicht in einem Gleichgewichtszustand verweilt, sondern diesen immer wieder von Neuem auf den Prüfstand hebt und überschreitet. Andernfalls, d. h. wenn das Leben >nur Organismus wäre, wäre es, mit Canguilhem gesprochen, pathologisch; stattdessen aber ist es Polarität und damit organisch und schöpferisch zugleich.

\section{VOM SOZIALEN ZUM VITALEN}

Ein solches Verständnis des Lebendigen in seiner radikalen Hierarchielosigkeit verabsolutiert jedoch, so könnte man etwas polemisch sagen, die Frage der vitalen Norm, die im Versuch zur Diskussion steht, und verschließt sich der Frage nach der Verfasstheit der sozialen Norm, insofern das Soziale einzig eine andere, jedoch der lebendigen Dynamik untergeordnete Dimension des Lebens ist. Eine spezifische Untersuchung der sozialen Norm lässt sich in diesem Rahmen genauso wenig vornehmen, wie eine spezifische Untersuchung des menschlichen Lebens. Dass auch Canguilhem dies als Desiderat empfindet, davon zeugt die Erweiterung seiner Reflexion auf die soziale Norm in den Neuen Überlegungen, die sowohl auf Foucaults Untersuchungen der Normen der »Klinik « als auch auf die Auseinandersetzung mit den Erkenntnissen der modernen Biologie zurückgehen, die der »Dynamik « des Lebens mit François Jacob eine »Logik « des Lebens entgegenstellen. ${ }^{18}$

Zugleich insistiert Canguilhem, wie bereits gesagt, zu Anfang der Neuen Überlegungen darauf, dass es das Ziel des von ihm nun ange- 
strengten Vergleichs »der gesellschaftliche[n] mit den Lebensnormen [ist], die spezifische Bedeutung der letzteren genauer zu fassen $« .{ }^{19}$ Canguilhem verbleibt also explizit innerhalb einer Analyse der vitalen Normen, die nun vor dem Hintergrund einer Analyse der sozialen Normen geschärft werden soll. Dieser weiterhin explizit vitalen Perspektive ist es geschuldet, dass Canguilhem die sozialen Phänomene ausschließlich unter dem Begriff der Normalisierung verhandelt - und nicht unter dem der Normativität -, womit markiert wird, dass das Soziale keinen Anteil hat an einer genuin normativen, also schöpferischen Dynamik:

So wie eine französische école normale eine Schule ist, auf der das Lehren gelehrt wird, d. h. Lehrmethoden experimentell unterrichtet werden, so ist ein Normaltropfenzähler jenes Gerät, das darauf geeicht ist, ein Gramm destilliertes Wasser in 20 frei fallende Tropfen zu teilen, damit so die pharmakodynamische Wirkkraft einer Lösungssubstanz genau nach ärztlicher Vorschrift abgestuft werden kann. ${ }^{20}$

Auch der standardisierte Abstand der europäischen Eisenbahnschienen wird als Normalspur bezeichnet. Während »das Normale « in all diesen Phänomenen als Resultat einer dem Phänomen selbst externen Entscheidung oder Auflage erscheint, weicht Canguilhems letztes Beispiel - die Bestimmung des Normalgewichts des Menschen als zu seiner Lebensdauer direkt korrelatives und damit artspezifisches Element - von dieser Relation ab und renormativiert die Normalisierung. Hier, so Canguilhem, entspringt das Normale dem Phänomen selbst - es entspricht einer Anforderung des Menschen auf eine lange Lebensdauer. In diesem Sinne und bei genauerem Hinsehen, sind jedoch auch die Beispiele der »Normung der technischen Mittel für Erziehung, Gesundheit und Transport [...] ein Ausdruck kollektiver Forderungen «, ${ }^{21}$ d. h., dass in ihnen das Streben einer Gesellschaft nach ihrem eigenen Wohl, dem bestmöglichen sozialen Gesamtzustand zum Ausdruck kommt.

Ein wesentliches Merkmal der Normalität, die Canguilhem hier anhand der "allerkünstlichsten Normung, nämlich der technischen « untersucht, ist es also, eine Relation bzw. eine Ko-Relativität zwischen Normen innerhalb eines Systems herzustellen. Die Frage bleibt jedoch, wie genau diese Verbindung, also letzten Endes die Herstellung eines Ganzen oder eines Kollektivs, zu verstehen ist. In dieser Hinsicht lässt sich der Unterschied zwischen sozialen und vitalen Normen folgendermaßen auf den Punkt bringen: Während die vitalen Normen in ihrem Zusammenspiel eine (organische) Totalität bilden, deren Teile immanent 
aufeinander abgestimmt sind und deren Regeln ohne Absicht und Berechnung wirken, bildet die Kohärenz der sozialen Norm ein Dispositiv oder Diagramm aus. ${ }^{22}$ Canguilhem selbst fasst diesen Gegensatz anhand der terminologischen Gegenüberstellung von (sozialer) Organisation und (vitalem) Organismus: In einer sozialen Organisation sind jene Normen oder Regeln, nach denen sich die Teile (d. h. die Teilnehmer an der Gesellschaft, die sozialen Partner) innerhalb eines sich über seine eigene Bestimmung bewussten Ganzen zusammenfügen, diesem sozialen Ganzen extern und müssen somit »vergegenwärtigt [représentées], gelernt, ins Gedächtnis zurückgerufen und angewendet werden « ${ }^{23}$ - das Soziale ist ein problematisches Ganzes; im Gegenteil dazu bildet die »Ordnung des Lebens [...] einen Zusammenhang von problemlos gelebten Regeln «, ${ }^{24}$ denn die Normen des lebendigen Organismus sind diesem immanent, sie müssen nicht angewandt oder dargestellt werden, sondern sind von sich aus aktiv - es gibt also in einer vitalen Ordnung keinen Bruch zwischen der Regel und der Regulierung. Der Unterschied besteht derart nicht in der homöostatischen Funktionsweise der Systeme, seien sie lebendig oder sozial, sondern in der Position und dem Ursprung der Normen in Bezug auf das Ganze, das von ihnen reguliert wird.

Die sozialen Normen, wie sie Canguilhem am Beispiel sprachlicher, industrieller und hygienischer Normen untersucht, werden mithin interessegeleitet, zum Wohle der Gesellschaft, festgesetzt, d. h. diese Festsetzung hängt von sozialen, politischen und ökonomischen Interessen und Bedingungen ab. Es ist gerade diese Tatsache, die für Canguilhem die sozialen von den vitalen Normen unterscheidet: Im Fall des Vitalen bezieht sich das, was durch die Norm reguliert wird - lebendige Prozesse und Organismen -, von selbst auf die Norm. Im Bereich des Sozialen hingegen muss der Gegenstand der Norm allererst unter die Norm gebracht bzw. auf sie bezogen werden, auch wenn es sich hierbei um allerseits "konsensfähige « Fragen des Allgemeinwohls handelt. ${ }^{25}$ Die Tatsache, dass die soziale im Gegensatz zur vitalen Norm nie problemlos gelebt wird, beruht also darauf, dass die Organisation der sozialen Normen nur scheinbar eine Synergie produziert.

Um diesen Schein zu überwinden, d. h. um die Verbindung von Sozialem und Vitalem als zwei inkommensurable Regimes oder »Gangarten [allures] « des Lebens herzustellen, stellt Canguilhem die sozialen und vitalen Normen in mehreren Passagen seines Textes in ein mimetisches Verhältnis und liefert damit, so die These, einen Einsatz für Fou- 
caults Ausformulierung biopolitischer bzw. gouvernementaler Praktiken, die sich nicht nur auf das Leben als ihren Gegenstand beziehen, sondern zugleich dessen innere Dynamik nachahmen.

DIE MIMETISIERUNG DES VITALEN DURCH DAS SOZIALE

Laut Canguilhem muss die gesellschaftliche Ordnung also als eine Art Synthese bzw. als ein Mischverhältnis beschrieben werden, insofern sie einerseits Teil hat an der organischen Ordnung: Sie ist "Schaffung von Organen [invention d'organes] «, ${ }^{26}$ insofern sie danach strebt, das Zusammenspiel ihrer Normen in einer homöostatischen, sich selbst regulierenden Ordnung zu verankern. Zugleich ist sie jedoch andererseits Teil der mechanischen Ordnung, da zwar ihre Normen organisch aufeinander abgestimmt sind, ihr Ziel oder die Richtung ihrer regulierenden Aktivität jedoch nicht aus der gesellschaftlichen Ordnung heraus, d. h. als Antwort auf ein immanentes Bedürfnis, produziert werden - vielmehr sind die »Zwecke der Gemeinschaft geplant «. ${ }^{27}$ In diesem Sinne schlussfolgert Canguilhem: »Wir können mit anderen Worten [...] sagen, dass eine Gesellschaft Maschine und Organismus ineins ist. ${ }^{28}$

Diese Synthese mechanischer und organischer Bestimmungen untersucht Canguilhem anhand des Modells der Imitation oder Mimesis des Vitalen durch das Soziale: Er formuliert diese Einsicht im Sinne einer Tendenz der sozialen Regulierung, das Modell der organischen Regulierung nachzuahmen, wobei er das Zusammenspiel der Elemente der sozialen Regulierung als mechanistisch beschreibt: "Die gesellschaftliche Regulation nähert sich also der organischen an und imitiert sie, bleibt aber dennoch mechanisch zusammengesetzt. « ${ }^{29}$ An anderer Stelle schreibt er im selben Sinne: »Die Phänomene der gesellschaftlichen Organisation sind gleichsam die Nachahmung der Lebensorganisation ähnlich wie Aristoteles die Kunst als Nachahmung der Natur begreift. Nachahmen heißt hier nicht kopieren, sondern den Sinn einer Produktion wieder finden. ${ }^{30}$

Die Funktionsweise sozialer Phänomene kann also als Nachahmung der vitalen Organisation verstanden werden, jedoch kopiert die gesellschaftliche Organisation nicht einfach die vitale, sondern wiederholt sie in jenem spezifischen Sinne, der ein Verständnis der vitalen Organisation voraussetzt und so ihre Mimetisierung im Rahmen der sozialen Möglichkeiten zulässt. Dabei wird weder der Unterschied zwischen Natürlichem und Künstlichem aufgehoben, noch vergessen, dass 
die sozialen Organisationen sich nicht von selbst immanent aus- und weiterbilden, so wie es die Organismen dank der Koordination ihrer natürlichen Kausalitäten tun. Für Canguilhem ist die Gesellschaft »ein Ensemble unzureichend vereinheitlichter Mittel gerade deswegen [...], weil ihr ein Zweck fehlt, mit dem sich die kollektive Aktivität identifizieren könnte «. ${ }^{31}$ Der Gesellschaft geht eine innere Normativität ab, sie hat keine immanente Norm, die ihre Anstrengungen in eine einheitliche Richtung lenken könnte und sie zugleich unmittelbar verständlich und erkennbar machen würde. Und trotzdem tendieren die sozialen Normen dazu, die organische Dynamik der vitalen Normen aufzunehmen, sie zu imitieren und in der Künstlichkeit des gesellschaftlichen Zusammenhangs zu veräußern: Die sozialen Regierungsformen verlieren derart zwar niemals ihren mechanischen Charakter, da sie dem zu regierenden Phänomen immer äußerlich bleiben, sie operieren aber zugleich in einer Als-ob-Logik und zwar als seien sie vitale Normen, insofern sie auf die Aufrechterhaltung homöostatischer Bevölkerungszustände abzielen.

Offen bleibt hier jedoch die Frage, inwiefern dieser homöostatischorganische Aspekt, den Canguilhem in seinen Texten zur sozialen Norm in den Vordergrund rückt (und der ehemals als pathologisch eingestuft wurde), mit einer genuin vitalen Dimension vereinbar ist. Das heißt, anders gesagt, ob der doppelte Lebensbegriff, den Canguilhem als zugleich selbsterhaltende und selbstüberschreitende Dynamik entwickelt, in dieser Wende tatsächlich verabschiedet wird, wie viele Kritiker und Leser Canguilhems es behaupten; oder ob vielmehr eine soziale Normativität denkbar wird, die das Soziale nicht einzig auf Normalisierung reduzieren würde. Das hieße, eine dritte Dimension in die Untersuchung der sozialen Norm bzw. des sozialen Leben (wieder) einzuführen, laut derer das soziale Leben Anteil am schöpferischen Prozess der Normen hat. Canguilhem selbst tut dies auf indirekte Weise, insofern er unterstreicht, dass, ganz gleich, ob das durch die Norm bestimmte »Normale « aus einer inneren Dynamik oder äußeren Setzung entspringt, seine Besonderheit darin liegt, dass es selbst wiederum zum Bezugspunkt oder »Ideal « für diejenigen Phänomene herhalten kann, die nicht, oder noch nicht, normal sind: »Das Normale bedeutet mithin sowohl Verbreitung wie Demonstration der Norm. « ${ }^{32}$ Das Normale egal welchen Ursprungs bildet folglich seine eigene Dynamik aus, indem es als Ideal für das Nicht-Normale fungiert: Die Norm erhält ihren Sinn (oder ihre Kraft) in der Konfrontation mit demjenigen, was sich ihr (noch) entzieht. Pierre Macherey spricht in diesem Zusammenhang von 
einer Analogie der Funktionsweisen vitaler und sozialer Normen, die jeweils in jenen Phänomenen oder Zuständen, die sich ihnen entziehen, ihre normative Aufforderung sehen. ${ }^{33}$ Vor diesem Hintergrund ist das »Normale «, so die berühmte Schlussfolgerung Canguilhems, »kein statischer und friedlicher Begriff, sondern ein dynamischer und polemischer « ${ }^{34}$

Eine Norm ist demnach für Canguilhem ein »möglicher Modus der Vereinheitlichung von Verschiedenem, der Integration von Unterschieden, der Beilegung von Differenzen ${ }^{35}$ und sie unterscheidet sich vom Naturgesetz, insofern sie keine notwendige, sondern ausschließlich eine mögliche Wirkung hat: Eine mögliche Wirkung, der andere mögliche Wirkungen entgegengesetzt oder an die Seite gestellt werden können. Damit läge die Dynamik bzw. der produktive Aspekt der sozialen Norm, analog zur vitalen Normativität, darin begründet, dass sie Abweichungen zu den bestehenden Normen produzieren kann und ihnen andere Normen entgegensetzt. Oder: In der Produktion von Normalität lässt sich ein neuer Individualisierungsprozess bestimmen, der andere soziale Normen schafft.

In diesem Sinne kann man schlussfolgern, dass das Soziale einer neuen Lebensform korrespondiert, die nicht dem vitalen Leben untergeordnet ist, sondern vielmehr derselben Normativität folgt, die durch Abweichungen neue Normen und Werte schafft. Denn das soziale System, insofern es gerade nicht durch Naturgesetze, sondern durch Normen organisiert und zusammengehalten wird, lässt, wie Canguilhem schreibt, Möglichkeiten offen, andere Normen zu schaffen und so in das soziale Dispositiv einzugreifen. So produziert die Normalisierung zwar eine Normalität im Sinne homöostatischer Rationalisierung, zugleich eröffnet sie jedoch auch eine normative Perspektive durch die Möglichkeit, abweichende (soziale) Normen zu schaffen, so wie es das Lebendige in seinem Milieu tut.

\section{CANGUILHEMS VITALISMUS}

Zusammenfassend kann man also sagen, dass die Wende vom Vitalen zum Sozialen in Canguilhems Denken zur Folge hat, dass der ehemals klar umrissene vitale Lebensbegriff an Eindeutigkeit verliert und sich in ein Mischverhältnis begibt zwischen organischen und mechanischen Bestimmungen, deren gegenseitige Durchdringung sich anhand des Begriffs der Technik ausformulieren lässt. ${ }^{36}$ Daraus folgt auch, dass die 
Frage nach der schöpferischen Dynamik des Lebens sich ebenfalls für das soziale Leben stellt, insofern dieses nicht einfach nur auf Prozesse der Normalisierung (bzw. Normierung) zu reduzieren ist, sondern auch genuin normative, also Normen schaffende Dynamiken umfasst.

Was also auch in diesem Übergang der 1960er Jahre infrage steht, ist der viel diskutierte Vitalismus Canguilhems: So verabschiedet beispielsweise Élisabeth Roudinesco Canguilhems Vitalismus in dieser theoretischen »Wende" der 1960er Jahre, d. h. im Übergang von einem vitalistischen Verständnis einer Norm, die »aus dem Leben kommt ", zum post-vitalistischen Verständnis der sozialen Norm, die sich in der bewussten Auseinandersetzung mit ihrem Ursprung in der Negation ausdifferenziert. ${ }^{37}$ Dies ist jedoch insofern zu einfach, als der infrage stehende »jugendliche "Vitalismus Canguilhems in einer solchen Lektüre zumeist jenseits jedweder Negativität als reine Affirmation eines Lebensprinzips verstanden wird. Allein, dieser Annahme ist Canguilhem bereits 1946-47 entgegen gegangen, wenn er in seinem Text "Aspekte des Vitalismus « mit dem klassischen Vitalismus eines Lebensprinzips bricht, zugleich jedoch mit der Formulierung des »permanenten Oszillierens des Lebens « einem reformulierten Vitalismus stattgibt. ${ }^{38}$

Dieser Vitalismus, der für Canguilhem »eher eine Moral als eine Theorie «, ein " permanenter Anspruch des Lebens im Lebendigen « ist, ${ }^{39}$ unterscheidet sich von animistischen oder naturalistischen Bestimmungen desselben, die Canguilhem als metaphysisch verwirft. Zugleich, und daran erinnert Monica Greco in ihrer Diskussion des Canguilhemschen Vitalismus zurecht, verwirft Canguilhem die ontologische Perspektive auf das Leben, für die der Vitalismus einsteht, nicht. Greco unterscheidet vielmehr zwei Verständnisse von Vitalismus bei Canguilhem: Erstens eine epistemologische Herangehensweise, die Foucault im Anschluss an Canguilhem unter dem Schlagwort des »theoretischen und kritischen Indikators ${ }^{40}{ }^{40}$ gefasst hat, und mit der Greco hier die Rekurrenz des Vitalismus " as a symptom of the specificity of life ${ }^{41}$ beschreibt. Neben dieser epistemologischen Situierung verweist der Vitalismus bei Canguilhem jedoch zweitens auch auf die Frage der Ontologie des Lebens - eine paradoxe Ontologie, die Greco als eine Ontologie der Kontingenz, »of what is permanently suspended between being and non-being « ${ }^{42}$ versteht, und die bei Foucault zu der Beschreibung des Canguilhemschen Denkens als einer »Philosophie des Irrtums « führt. Denn das Leben selbst ist ein instabiles, kontingentes, irrtumsanfälliges und vermischtes Leben, das sich zwischen mechanischen, organisch-erhaltenden und 
vitalistisch-schöpferischen Bestimmungen ausbildet: Das Leben wird aus Zuständen des Lebens und des Nicht-Lebens hervorgebracht. Dies zeigt sich in der so genannten Wende der 1960er Jahre und dies ist es auch, was den Bezug Foucaults auf eine solche Ontologie des Lebens überhaupt möglich macht und den zentralen Impuls für die Rolle liefert, die der Lebensbegriff als Korrelat von Macht- und Wissenstechniken in Foucaults Schriften einnimmt.

In diesem Sinne spricht auch Frédéric Worms von dem »tiefgreifend erneuerten, aber auch bejabten [profondément assumé] >Vitalismus Canguilhems «, ${ }^{43}$ der sich in dem neuen philosophische Moment, den die 1960er Jahre darstellen, zwei Herausforderungen stellen muss, einer epistemologischen (die genetische Revolution) und einer historisch-politischen (die soziale Norm). Worms beschreibt Canguilhems Wende jedoch zugleich als »Fortführung und Verschiebung (maintien et décalage) «, die sich in epistemologischer Hinsicht folgendermaßen fassen lässt:

Der Beweis für die unüberwindbare Normativität des »Lebendigen« liegt nicht mehr im Gegenstand der Erkenntnis, sondern in ihrem Subjekt, d. h. in seinen Praktiken und seiner Geschichte. Obgleich das »Leben« de jure Gegenstand eines objektiven Wissens ist und sogar auf objektiven Aprioris - wie dem genetischen Code - begründet ist, wird die Erkenntnis dieser Aprioris immer die polarisierte Aktivität eines menschlichen Lebewesens sein. $^{44}$

Selbst wenn das biologische Leben durch den genetischen Code determiniert wird (und in diesem Sinne nicht mehr normativ zu nennen wäre), wird die Erkenntnis selbst als eine polarisierte Aktivität verstanden und damit als Realisierung der (sozialen) Normativität des Menschen lesbar. Die historisch-politische Problematisierung dieses Denkens findet ihre Ausformulierung in Foucaults Untersuchungen zur Klinik und dem »medizinischen Blick «, die Worms nun als eine andere Art von »Normen « beschreibt, die der biologisch-vitalen Normativität »en droit et en fait « vorgängig sind. Hierbei handelt es sich um die zugleich epistemologische und politisch-praktische Normativität der Diskurse und Aussagensysteme, die das Leben, ja sogar das »Leben als Polarität ", zu ihrem Objekt bzw. zu ihrem Effekt, ihrer Wirkung machen:

Es ist unter dem klinischen Blick, unter der Bedingung dieses Blicks und dieses Diskurses, dass sich die Polarität des Lebendigen und des Todes (in der Leiche selbst) eröffnet, und es ist nicht in der Polarität des Normalen 
und des Pathologischen, dass sich die Möglichkeit und die Notwendigkeit der klinischen Erfahrung eröffnet. ${ }^{45}$

Das heißt, dass in dieser Wendung nicht etwa der vitale Lebensbegriff aufgegeben wird oder Canguilhem sich vom Vitalismus abwendet, sondern dass die eigenartige Verfasstheit des Lebens als Mischwesen oder Synthese aus vitalen und sozialen, organischen und mechanischen, inneren und äußeren Bestimmungen selbst thematisch wird als etwas, das im Diskurs, oder in den Macht-Wissens-Techniken hervorgebracht - synthetisiert - wird. Diese Vermischung, die sich einerseits anhand des Begriffs der Technik ausformulieren lässt, ist andererseits zentral für die hier vertretene These, Foucaults biopolitische Techniken würden sich in einem (im Anschluss an Canguilhem verstandenen) mimetischen Bezug zu einem Leben befinden, das selbst als ein synthetischer Begriff in Erscheinung tritt. Denn das Leben, daran erinnert Foucault am eingängigsten in Die Ordnung der Dinge, ist ein moderner bzw. ein im doppelten Sinne synthetischer Begriff, insofern einerseits seine von der Biopolitik aufgenommene biologische Dynamik auf die Formierung eines synthetischen Ganzen abzielt, d. h. eines globalen Gleichgewichts, das in der Bevölkerung seine Gestalt findet, und das Leben andererseits "als Leben « synthetisiert, also hergestellt ist. Es ist ein gemachtes Phänomen und wird in dieser biopolitisch-ökonomischen Perspektive als eine artifiziell durchorganisierte Natur verstanden.

\section{BIOPOLITIK ALS IMITATION DES LEBENS}

In diesem Sinne kann der Lebensbegriff bei Canguilhem und seine vitale Dynamik für das Verständnis sozialer Normen fruchtbar gemacht werden, d. h. es kann eine soziale Normativität gedacht werden, die in doppeltem Sinne den machtanalytischen Untersuchungen Foucaults stattgibt, insofern sie einerseits die Untersuchungen zu Biopolitik und Gouvernementalität informiert - denn die Biopolitik funktioniert, im Gegensatz zur Disziplin und der souveränen Macht, wie eine soziale Dynamik bzw. Normativität - und andererseits eine Perspektive auf das synthetische Leben - also auf die Gemachtheit oder Künstlichkeit desselben - eröffnet, die das Leben nicht als Essenz, sondern als Korrelat, d. h. ausschließlich in seiner Durchsetzung von Macht- und Wissensstrategien versteht, von denen es zuallererst hergestellt wird. Diese zweite Perspektive, so die These, ist in Canguilhems vitalistischen Unter- 
suchungen des Lebens insofern angelegt, als hier die Durchdringung, Mimetisierung oder Synthetisierung vitaler und sozialer Phänomene, Normen oder Perspektiven im Leben denkbar wird.

Canguilhems Ontologie des Lebens eröffnet damit eine Philosophie des Irrtums, d. h. ein Verständnis des Lebens als von Außen, in Auseinandersetzung mit seinen negativen Werten produziertes. Diese Ontologie wird von Foucault als biopolitische Produktionsweise ausgeflaggt: Das Leben erscheint bei Foucault also dezidiert nicht als ontologisches, vielmehr werden im Gegenteil die Wissensmechanismen und Machttechniken untersucht, die einen solchen Lebensbegriff - als »archäologisches Oberflächenphänomen « - produzieren oder hervorrufen. Damit bezeichnet der Foucaultsche Lebensbegriff ein synthetisiertes Leben, dessen Ursprung sich jedoch bei Canguilhem wiederfindet, insofern er das »soziale « Leben als einen Mischzustand zwischen mechanischen und organischen, künstlichen und natürlichen Bestimmungen, erster und zweiter Natur beschreibt. So stellt Foucault eine Machttheorie bereit, deren Funktionsweise in der Projektion, Produktion und Imitation einer solchen lebendigen Gemengelage besteht. Folglich bezieht sich die Biopolitik nicht auf ein gegebenes, dynamisches Leben - vielmehr bringt sie es als pseudo-lebendiges zuallererst hervor. Damit funktioniert die gouvernementale Biopolitik als eine Art Illusionsmaschine, die das »Leben als Polarität « im Leben der Bevölkerung synthetisiert und im künstlich-natürlichen Milieu der gouvernementalen Techniken regiert. ${ }^{46}$ Foucaults Begriff der Biopolitik der Bevölkerung als einer Macht, die das Leben durchdringt, und die daran anschließende Formulierung der Gouvernementalität als Verschaltung von Selbst- und Fremd-Reg(ul)ierung erschließt sich also erst, wenn man seine Anlehnung an die Canguilhemsche Figur der Imitation berücksichtigt: Die sozial verfasste Sicherheitsnorm imitiert die vitale Dynamik des Lebendigen und überträgt bzw. veräußert sie auf einer machttheoretischen und -praktischen Ebene. Ein zeitgenössisches politisches Wissen im Anschluss an Canguilhem ist derart in diesem Verhältnis, d. h. in der Verflechtung von vitalen und sozialen Normen, von natürlichen und künstlichen Prozessen verankert, bzw. in der Synthese, der sie - im Begriff des Lebens - stattgeben. 


\section{A N M ER K U N GE N}

1 Georges Canguilhem, Das Normale und das Pathologische (Frankfurt a.M.: Ullstein, 1977), S. 161.

2 Georges Canguilhem, Die Erkenntnis des Lebens, übers. v. Till Bardoux, Maria Muhle u. Francesca Raimondi (Berlin: August Verlag, 2009).

3 Xavier Bichat, Recherches physiologiques sur la vie et la mort (Paris: Flammarion, 1994), S. 57.

4 Canguilhem, »Das Denken und das Lebendige", in ders., Die Erkenntnis des Lebens, S. 21.

5 Monica Greco erinnert daran, dass Canguilhems Originalität des Lebens als logische Priorität des Lebens vor dem Wissen vom Leben, also der Wissenschaft, verstanden werden muss. So schreibt Canguilhem: »Wenn man die Originalität des Lebens anerkennt, muss man die Materie innerhalb des Lebens und die Wissenschaft der Materie, das heißt die Wissenschaft schlechthin, innerhalb der Aktivität des Lebendigen >begreifen< [comprendre] « (Canguilhem, "Aspekte des Vitalismus ", in ders., Die Erkenntnis des Lebens, S. 149-81, hier S. 173). Das heißt, dass der Standpunkt des Lebens keine Ausnahme hinsichtlich des physikalischen Milieus bilden kann, so wie es der klassische, animistische oder naturalistische Vitalismus annimmt, sondern diesem vorgängig ist. Vgl. Monica Greco, »On the Vitality of Vitalism", Theory, Culture \& Society, 22.1 (2005), S. 15-27, hier S. 19.

6 Canguilhem, »Das Lebendige und sein Milieu «, in ders., Die Erkenntnis des Lebens, S. 233-79, hier S. 260 (Hervorhebung M.M.).

7 Canguilhem, Das Normale und das Pathologische, S. 121. Das Zitat stammt von Robert Reininger, Wertphilosophie und Ethik (Wien: Braunmüller, 1939), S. 29 (Hervorhebung M.M.).

8 Canguilhem, Das Normale und das Pathologische, S. 89.

9 Georges Canguilhem, "Le concept et la vie «, in ders., Études d'histoire et de philosophie des sciences (Paris: Vrin, 1968), S. 350 (Übersetzung M.M.).

10 Canguilhem, »Das Lebendige und sein Milieu«, S. 264-65 (Hervorhebung M.M.).

11 Das Leben ist, so Canguilhem Walter Cannon paraphrasierend, »ein Zusammenhang fragiler und gefährdeter, aber durch ein Regulationssystem ständig wiederhergestellter Funktionen ". (Canguilhem, Das Normale und das Pathologische, S. 180.)

12 Während Canguilhem von Goldstein den Begriff der organischen Regulation übernimmt, geht er in der Bestimmung einer schöpferischen Kraft des Lebens über das Biologieverständnis Goldsteins hinaus. Die organischen Normen werden sowohl durch die Regulation ihres inneren Gleichgewichts als auch durch die Aufrechterhaltung einer ausgeglichenen Beziehung zwischen dem Leben und der Umwelt (normes de centration) bestimmt. Hier folgt Canguilhem den Thesen Goldsteins. Im Hinblick auf die Schöpfung der Normen setzt er sich jedoch von ihnen ab, denn Goldstein denkt diese Schöpfung ausschließlich innerhalb eines homöostatischen Ganzen, d. h. innerhalb einer organischen Struktur, die als Ganze den Gesetzen der Konstanthaltung untersteht. Die organische Norm ist 
die Setzung einer solchen organischen Konstanz, die zugleich die Struktur des organischen Verhaltens (der Selbsterhaltung) liefert. Canguilhems Verständnis der Norm geht über diese Position hinaus, insofern für ihn die Schöpfung der Normen nicht der Regel der Konstanthaltung unterworfen sein kann, sondern sich mit dieser in einer produktiven und permanenten Auseinandersetzung befindet. Dies ist der zweite Sinn, den die Normativität bei Canguilhem gewinnt, und der im folgenden Paragraph erläutert wird.

13 Georges Canguilhem, »Régulation ", in Encyclopæedia Universalis (Paris: Edition Encyclopædia Universalis, 1989), S. 712 (Übersetzung M.M.).

14 Canguilhem, »Das Lebendige und sein Milieu «, S. 265 (Hervorhebung M.M.).

15 Guillaume Le Blanc, Canguilhem et les normes (Paris: PUF, 1998), S. 55 (Übersetzung und Hervorhebung M.M.). Zugleich subjektiviert es sich - und damit wäre bereits hier eine soziale Dimension eingeführt, die sich jedoch der lebendigen Dynamik unterordnet bzw. keine spezifischen Unterschiede zu ihr aufweist im Bewusstsein dieser Individualisierung.

16 »Wenn man nach Maßgabe der Physiologie von einem normalen Menschen sprechen kann, so deswegen, weil es normative Menschen gibt, das heißt Menschen, für die es normal ist, die geltenden Normen zu sprengen und neu in Kraft zu setzen.« (Canguilhem, Das Normale und das Pathologische, S. 110.)

17 Ebd., S. 155.

18 Vgl. François Jacob, Die Logik des Lebenden. Eine Geschichte der Vererbung (Frankfurt a.M.: Fischer, 2002) und Foucaults enthusiastische Rezension zur Veröffentlichung des Buches in Frankreich: Michel Foucault, "Wachsen und Vermehren ", in ders., Schriften in vier Bänden. Dits et Ecrits, hg. v. Daniel Defert u. François Ewald (Frankfurt a.M.: Suhrkamp, 2001-05), II: 1970-1975 (2002), S. 123-28.

19 Canguilhem, Das Normale und das Pathologische, S. 161.

20 Ebd., S. 162.

21 Ebd.

22 Vgl. Le Blanc, Canguilhem et les normes, S. 86.

23 Canguilhem, Das Normale und das Pathologische, S. 172.

24 Ebd.

25 So wie dies beispielsweise der Fall ist, wenn die westlichen Mächte ihr höchstes politisches Gut, die Demokratie, in die Länder der so genannten zweiten oder dritten Welt exportieren, und diese demokratische Norm wenn nötig auch mit Waffengewalt durchsetzen, indem sie den Gegenstand, in diesem Fall die als nicht-demokratisch eingestuften Bevölkerungen, unter diese Norm bringen. Vgl. zu dieser Diskussion u. a. Jacques Rancière, Der Hass der Demokratie (Berlin: August Verlag, 2011) und ders., "Wer ist das Subjekt der Menschenrechte", in Die Revolution der Menschenrechte, hg. v. Christoph Menke u. Francesca Raimondi (Frankfurt a.M.: Suhrkamp, 2011), S. 474-90.

26 Canguilhem, Das Normale und das Pathologische, S. 175.

27 Ebd., S. 174.

28 Ebd. (Hervorhebung M.M.).

29 Ebd., S. 177 (Hervorhebung M.M.). 
30 Ebd., S. 175.

31 Ebd., S. 177 (Übersetzung leicht abgeändert M.M.).

32 Canguilhem, Das Normale und das Pathologische, S. 163.

33 Vgl. Pierre Macherey, "Normes vitales et normes sociales ", in Actualité de Georges Canguilhem, hg. v. François Bing, Jean-François Braunstein u. Elisabeth Roudinecso (Paris: Synthélabo, 1998), S. 71-84.

34 Canguilhem, Das Normale und das Pathologische, S. 163.

35 Ebd., S. 164.

36 Siehe hierzu u. a. Georges Canguilhem, Wissenschaft, Technik, Leben. Beiträge zur historischen Epistemologie, übers. v. Ronald Vouillé u. a., hg. u. mit einem Nachwort v. Henning Schmidgen (Berlin: Merve, 2006).

37 Vgl. Élisabeth Roudinesco, »Georges Canguilhem, de la médecine à la résistance. Destin du concept de normalité", in Actualité de Georges Canguilhem, hg. v. Bing, Braunstein u. Roudinecso, S. 13-41, hier S. 39.

38 Canguilhem, "Aspekte des Vitalismus", in ders., Die Erkenntnis des Lebens, S. 149-81, hier S. 153. Siehe für eine eingehende kritische Diskussion von Canguilhems Bestimmung des Lebens als "permanentes Oszilllieren « bzw. als »dialektische Essenz « Christoph F. E. Holzhey, »Kippbilder des Vitalen. Lebenswissen bei Canguilhem und Haraway « im vorliegenden Band; siehe zur Frage des Vitalismus bei Canguilhem auch Maria Muhle, "Zweierlei Vitalismus «, in Philosophie und Nicht-Philosophie. Aktuelle Diskussionen, hg. v. Friedrich Balke u. Mark Rölli (Bielefeld: Transcript, 2011), S. 71-96.

39 Canguilhem, »Aspekte des Vitalismus «, S. 158.

40 Michel Foucault, »Vorwort von Michel Foucault « (Vorwort zu Georges Canguilhem, On the Normal and the Pathological, Boston 1978), in ders., Schriften in vier Bänden, III: 1976-1979 (2003), S. 551-67, hier S. 562.

41 Greco, »On the Vitality of Vitalism «, S. 20.

42 Ebd., S. 18.

43 Frédéric Worms, "La vie dans la philosophie du xx ${ }^{\text {ème }}$ siècle en France ", Philosophie(s) française(s), Philosophie, 109 (Paris: Editions de Minuit, 2011), S. 74-91, hier S. 83 (Hervorhebung, M.M.).

44 Ebd., S. 83-84. Zugleich beinhaltet auch die berühmte "Logik des Lebendigen «, die hier paradigmatisch für die durch die Entdeckung des genetischen Codes erfolgten Umwälzungen steht, Irrtümer bzw. Übertragungsfehler, wie Canguilhem es in seinem Text zum Irrtum ausführt. Diese Irrtümer stellen die Überzeugung in Frage, dass das Lebendige sich in seiner Auseinandersetzung mit dem Milieu ausbilden würde, da seine »Logik « schon im Vorhinein, durch den genetischen Code, festgelegt ist. (Vgl. hierzu Giuseppe Bianco, "Portées du nom >Bergson «, Philosophie(s) française(s), Philosophie, 109 [Paris: Editions de Minuit, 2011], S. 43-59, hier S. 51-52, Anm. 42.) Canguilhem verweist jedoch darauf, dass auch genetische Fehler zum Teil latent existieren und erst in Auseinandersetzung mit dem Milieu pathologisch werden. In diesem Zusammenhang diskutiert Macherey das von Canguilhem zitierte Beispiel eines Kindermädchens, das erst bei einer Reise in die Berge (d. h. in eine anderes, ungewohntes Milieu) feststellt, dass sie, unter bestimmten Umständen, aber nicht unter snormalen< Umständen, 
an Hypotonie leidet. Macherey wiederum macht hieran die interne Verbindung von sozialen und vitalen Normen fest, da die Tatsache, dass es eine Welt gibt, in der es Kindermädchen gibt, Sommerfrische und eine Lohnbeziehung zwischen bürgerlicher Familie und Dienstpersonal eine ausschließlich soziale Tatsache ist. Damit zeigt Macherey, dass schon 1943, in Canguilhems Versuch also, die "Situation ", in der das Kindermädchen in den Bergen an ihrer Hypotonie erkrankt, "est à la lettre surdétérminé par des conditions relevant de normes vitales et sociales" (Macherey, "Normes vitales et normes sociales", S. 82-83; Hervorhebung M.M.).

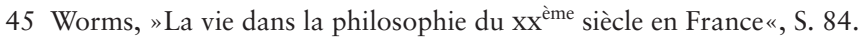

46 Vgl. hierzu Foucaults Untersuchungen zum künstlich-natürlichen Milieu der gouvernementalen Regierungstechniken, die er als Sicherheitsräume bezeichnet, in Michel Foucault, Sicherheit, Territorium, Bevölkerung, Biopolitik (Frankfurt a.M.: Suhrkamp, 2004), bes. S. 42-43. Vgl. auch Maria Muhle, Eine Genealogie der Biopolitik. Zum Begriff des Lebens bei Foucault und Canguilhem (Bielefeld: Transcript, 2008), S. 157-260. 
Maria Muhle, »Vom Vitalen zum Sozialen: Überlegungen zu einem politischen Wissen im Anschluss an Canguilhem «, in Situiertes Wissen und regionale Epistemologie: Zur Aktualität Georges Canguilhems und Donna J. Haraways, hg. v. Astrid Deuber-Mankowsky und Christoph F. E. Holzhey, Cultural Inquiry, 7 (Wien: Turia + Kant, 2013), S. 141-59<https://doi.org/10.37050/ci-07_07>

\section{QUELLENANGABEN}

Bichat, Xavier, Recherches physiologiques sur la vie et la mort (Paris: Flammarion, 1994)

Canguilhem, Georges, Die Erkenntnis des Lebens, übers. v. Till Bardoux, Maria Muhle u. Francesca Raimondi (Berlin: August, 2009)

—Das Normale und das Pathologische, übers. v. Monika Noll u. Rolf Schubert (München: Hanser, 1974)

—Wissenschaft, Technik, Leben. Beiträge zur historischen Epistemologie, übers. v. Ronald Vouillé u. a., hg. u. mit einem Nachwort v. Henning Schmidgen (Berlin: Merve, 2006)

— $\gg$ Régulation «, in Encyclopeedia Universalis (Paris: Edition Encyclopædia Universalis, 1989)

Études d'histoire et de philosophie des sciences, 7. erw. Aufl. (Paris: Vrin, 1994)

Foucault, Michel, Schriften in vier Bänden. Dits et Ecrits, hg. v. Daniel Defert u. François Ewald (Frankfurt a.M.: Suhrkamp, 2001-05)

Sicherheit, Territorium, Bevölkerung, Biopolitik (Frankfurt a.M.: Suhrkamp, 2004)

Giuseppe Bianco, »Portées du nom $>$ Bergson $<\ll$, Philosophie(s) française(s), Philosophie, 109 (Paris: Editions de Minuit, 2011), S. 43-59 <https://doi.org/10.3917/philo.109.0043>

Greco, Monica, »On the Vitality of Vitalism«, Theory, Culture \& Society, 22.1 (2005), S. 15-27 $<$ https://doi.org/10.1177/0263276405048432>

Jacob, François, Die Logik des Lebenden. Eine Geschichte der Vererbung (Frankfurt a.M.: Fischer, 2002)

Le Blanc, Guillaume, Canguilhem et les normes (Paris: PUF, 1998)

Macherey, Pierre, »Normes vitales et normes sociales «, in Actualité de Georges Canguilhem, hg. v. François Bing, Jean-François Braunstein u. Elisabeth Roudinecso (Paris: Synthélabo, 1998), S. 71-84

Muhle, Maria, Eine Genealogie der Biopolitik. Zum Begriff des Lebens bei Foucault und Canguilhem (Bielefeld: Transcript, 2008)

Rancière, Jacques, Der Hass der Demokratie (Berlin: August, 2011)

»Wer ist das Subjekt der Menschenrechte «, in Die Revolution der Menschenrechte, hg. v. Christoph Menke u. Francesca Raimondi (Frankfurt a.M.: Suhrkamp, 2011), S. 474-90

Reininger, Robert, Wertphilosophie und Ethik (Wien: Braunmüller, 1939)

Roudinesco, Elisabeth, »Georges Canguilhem, de la médecine à la résistance. Destin du concept de normalité «, in Actualité de Georges Canguilhem, hg. v. Bing, Braunstein u. Roudinecso, S. 13-41

Worms, Frédéric, $\gg$ La vie dans la philosophie du $\mathrm{xx}^{\text {ème }}$ siècle en France $\ll$, Philosophie(s) française(s), Philosophie, 109 (Paris: Editions de Minuit, 2011), S. 74-91<https://doi.org/10. 3917/philo.109.0074> 